\title{
Radicals of ideals that are not the intersection of radical primes
}

\author{
by
}

\section{Laksov and M. Rosenlund (Stockholm)}

\begin{abstract}
Various kinds of radicals of ideals in commutative rings with identity appear in many parts of algebra and geometry, in particular in connection with the Hilbert Nullstellensatz, both in the noetherian and the non-noetherian case.

All of these radicals, except the $\star$-radicals, have the fundamental, and very useful, property that the radical of an ideal is the intersection of radical primes, that is, primes that are equal to their own radical. It is easy to verify that when the ring $A$ is noetherian then the $\star$-radical $R(\mathfrak{I})$ of an ideal is the intersection of $\star$-radical primes. However, it has been an open question whether this holds in general. The main purpose of this article is to give an example of a ring with a $\star$-radical that is not radical. To our knowledge it is the first example of a natural radical on a ring such that the radical of each ideal is not the intersection of radical primes. More generally, we present a method that may be used to construct more such examples.

The main new idea is to introduce radical operations on the closed sets of topological spaces. We can then use the Zariski topology on the spectrum of a ring to translate algebraic questions into topology. It turns out that the quite intricate algebraic manipulations involved in handling the $\star$-radical become much more transparent when rephrased in geometric terms.
\end{abstract}

Introduction. Various kinds of radicals of ideals in commutative rings with identity appear in many parts of algebra and geometry (see [B], [BCR], $[\mathrm{BGN}],[\mathrm{E}],[\mathrm{L} 1]$ for material relevant to the present work), in particular in connection with the Hilbert Nullstellensatz, both in the noetherian and the non-noetherian case (see [BCR], [K1], [K2], [L2], [R1], [R2]).

All of these radicals, except the $\star$-radicals, have the fundamental, and very useful, property that the radical of an ideal is the intersection of radical primes, that is, primes that are equal to their own radical (see Examples 1.3 and 2.2). The $\star$-radical of a ring $A$ corresponds to an operation $R$ on ideals

2000 Mathematics Subject Classification: 13A10, 13A15, 14A05, 14A15, 54B35.

Key words and phrases: quasi-radicals, radicals, quasi-radicals and radicals on topological spaces, Zariski topology, non-noetherian rings, Hilbert Nullstellensatz. 
in the ring that satisfies the following attractive set of axioms (see [BGN] and $[\mathrm{B}])$ :

For all ideals $\mathfrak{I}$ and $\mathfrak{J}$ of $A$ :

(1) $\mathfrak{I} \subseteq R(\mathfrak{I})$.

(2) $R(R(\mathfrak{I}))=R(\mathfrak{I})$.

(3) $R(\mathfrak{I} \mathfrak{J})=R(\mathfrak{I} \cap \mathfrak{J})=R(\mathfrak{I}) \cap R(\mathfrak{J})$.

It is easy to verify that when the ring $A$ is noetherian the $\star$-radical $R(\mathfrak{I})$ of an ideal is the intersection of $\star$-radical primes. However, it has been an open question whether this holds in general. The main purpose of this article is to give an example of a ring with a $\star$-radical that is not radical. To our knowledge it is the first example of a natural radical on a ring such that the radical of some ideal is not the intersection of radical primes. More generally, we present a method that may be used to construct more such examples.

Since the main purpose of this article is to show that there exist rings with a $\star$-radical that contain $\star$-radical ideals that are not the intersection of $\star$-radical prime ideals, we have changed the terminology so that a $\star$-radical on a ring corresponds to what we call a quasi-radical operation on the ideals of the ring, and we call an operation radical when the radical of all ideals of the ring is the intersection of primes that are equal to their own radical.

The main new idea is to introduce radical operations on the closed sets of topological spaces. We can then use the Zariski topology on the spectrum of a ring to translate algebraic questions into geometry. It turns out that the quite intricate algebraic manipulations involved in handling the quasi-radical operations become much more transparent when rephrased in geometric terms. It is, for example, quite easy to show that the quasi-radical operation on any topological space that associates to a closed set the closure of its interior, when applied to the Zariski topology of a ring, gives a quasi-radical operation on the ring. With this quasi-radical we are able to prove that the polynomial ring $K\left[t_{1}, t_{2}, \ldots\right]$ in countably many independent variables over a field, modulo the ideal generated by the elements $t_{i}\left(t_{i}-1\right)$ for $i=1,2, \ldots$, has quasi-radical ideals that are not the intersection of radical primes.

In the correspondence between the ideals of a ring and the closed sets in the Zariski topology on the spectrum, ideals with the same radical give the same closed set. It is consequently quite astonishing that it is at all possible to have a notion of radicals for topological spaces.

Although it is not used in the article, it may illustrate the correspondence between algebra and topology to give an explicit expression for the quasiradical of an ideal $\mathfrak{I}$ in a ring $A$ that is given by the quasi-radical, on the spectrum $X=\operatorname{Spec}(A)$ with the Zariski topology, that to a closed subset 
associates the closure of its interior. Denote by $C(\mathfrak{I})$ the smallest ideal in $A$ that contains all ideals $\mathfrak{J}$ with the property that if $\mathfrak{p}$ is a prime ideal that does not contain $\mathfrak{I}$, then $\mathfrak{p}$ contains $\mathfrak{J}$. Let $\mathcal{V}(\mathfrak{J})=\{\mathfrak{p}: \mathfrak{p} \supseteq \mathfrak{J}\}$. Then, by definition, $C(\mathfrak{I})$ is the smallest ideal containing the ideals $\mathfrak{J}$ such that $\mathcal{V}(\mathfrak{J}) \supseteq \operatorname{Spec}(A) \backslash \mathcal{V}(\mathfrak{I})$. Denote, in any topological space, by $\bar{Z}$ and $Z^{0}$ the closure, respectively the interior, of a set $Z$. We use the general formula $\bigcap_{Z \text { closed, } Z \supseteq X \backslash Y} Z=\overline{X \backslash Y}$, valid for any closed set $Y$, to obtain

$$
\mathcal{V}(C(\mathfrak{I}))=\bigcap_{\mathcal{V}(\mathfrak{J}) \supseteq X \backslash \mathcal{V}(\mathfrak{I})} \mathcal{V}(\mathfrak{J})=\overline{X \backslash \mathcal{V}(\mathfrak{I})}
$$

That is, $C(\mathfrak{I})$ gives the set $\overline{X \backslash \mathcal{V}(\mathfrak{I})}$. From the general equality $\overline{X \backslash(\overline{X \backslash Y})}=$ $\overline{Y^{0}}$ we obtain

$$
\mathcal{V}(C C(\mathfrak{I}))=\overline{X \backslash(\overline{X \backslash \mathcal{V}(\mathfrak{I})})}=\overline{\mathcal{V}(\mathfrak{I})^{0}},
$$

so that $C C(\mathfrak{I})$ is the quasi-radical of $\mathfrak{I}$.

1. Quasi-radicals. In this section we define quasi-radical operations on ideals of rings and give their main properties (see also [B] and [BGN]). Proposition 1.4 is also proved in [B].

1.1. Notation. Let $A$ be a commutative ring with unit. For each ideal $\mathfrak{I}$ of $A$ we let $\sqrt{\mathfrak{I}}=\left\{f \in A: f^{n} \in \mathfrak{I}\right.$ for some positive integer $\left.n\right\}$ be the usual radical of $\mathfrak{I}$.

1.2. Definition. An operation $R$ on the ideals of $A$ is a correspondence that to every ideal $\mathfrak{I}$ of $A$ associates an ideal $R(\mathfrak{I})$ of $A$. We say that the operation $R$ is quasi-radical if it satisfies the following three conditions for all ideals $\mathfrak{I}$ and $\mathfrak{J}$ of $A$ :

$(\mathrm{QR} 1) \quad \mathfrak{I} \subseteq R(\mathfrak{I})$.

(QR2) $\quad R(R(\mathfrak{I}))=R(\mathfrak{I})$.

(QR3) $\quad R(\mathfrak{I} \mathfrak{J})=R(\mathfrak{I} \cap \mathfrak{J})=R(\mathfrak{I}) \cap R(\mathfrak{J})$.

Let $R$ be a quasi-radical operation on the ring $A$ and let $\mathfrak{I}$ be an ideal of $A$. We call $R(\mathfrak{I})$ the quasi-radical of $\mathfrak{I}$, and say that $\mathfrak{I}$ is quasi-radical if $R(\mathfrak{I})=\mathfrak{I}$.

1.3. ExAMPLES. (1) The usual radical is clearly a quasi-radical operation on the ideals of $A$. We shall show in Proposition 1.4 that $\sqrt{\mathfrak{I}} \subseteq R(\mathfrak{I})$ for all quasi-radical operations $R$. Hence the usual radical is the finest quasi-radical operation on the ideals.

(2) The coarsest quasi-radical operation is the operation defined by $R(\mathfrak{I})=A$ for all ideals $\mathfrak{I}$ of $A$.

(3) (See [BCR], or [R1] and [R2].) Let $A$ be a ring and let $M$ be a subset that is stable under addition and multiplication and such that $f^{2} \in M$ for 
all $f \in A$. For all ideals $\mathfrak{I}$ of $A$ we let

$$
R(\mathfrak{I})=\left\{f \in A \text { : there exist } m \in \mathbb{N} \text { and } g \in M \text { such that } f^{2 m}+g \in \mathfrak{I}\right\} .
$$

Then $R$ is a quasi-radical operation on ideals.

(4) (See [L1].) Let $K$ be a subfield of a field $L$. Moreover, let $t_{1}, t_{2}, \ldots$ be a countable number of independent variables over $K$, and let $K\left[t_{1}, t_{2}, \ldots\right]$ be the polynomial ring in these variables over $K$. For each non-negative integer $m$ let

$P_{K}(m)=\left\{p\left(t_{1}, \ldots, t_{m}\right): p\right.$ a homogeneous polynomial with coefficients in $K$ and if $p\left(k_{1}, \ldots, k_{m}\right)=0$ with $k_{1}, \ldots, k_{m}$ in $L$ then $\left.k_{m}=0\right\}$.

For each ideal $\mathfrak{I}$ of a ring $A$ we let $R(\mathfrak{I})=\left\{f \in A:\right.$ for some positive integer $m$ there are $p \in P_{K}(m)$ and $f_{1}, \ldots, f_{m-1}$ in $A$ such that $\left.p\left(f_{1}, \ldots, f_{m-1}, f\right) \in \mathfrak{I}\right\}$.

Then $R$ defines a quasi-radical operation.

1.4. Proposition. Let $R$ be a quasi-radical operation on the ring $A$, and let $\mathfrak{I}$ and $\mathfrak{J}$ be ideals of $A$. Then:

(1) If $\mathfrak{I} \subseteq \mathfrak{J}$ then $R(\mathfrak{I}) \subseteq R(\mathfrak{J})$.

(2) $R(\mathfrak{I})=R(\sqrt{\mathfrak{I}})=\sqrt{R(\mathfrak{I})}$.

Proof. (1) It follows from (QR3) that if $\mathfrak{I} \subseteq \mathfrak{J}$ then $R(\mathfrak{I})=R(\mathfrak{I} \cap \mathfrak{J})=$ $R(\mathfrak{I}) \cap R(\mathfrak{J})$. Hence $R(\mathfrak{I}) \subseteq R(\mathfrak{J})$.

(2) For every $f$ in $A$ it follows from (QR3) that $R((f))=R((f) \cap(f))=$ $R\left(\left(f^{2}\right)\right)$. By induction on $n$ we obtain $R\left(\left(f^{n}\right)\right)=R((f))$ for all positive integers $n$.

Let $f \in \sqrt{R(\mathfrak{I})}$. Then $f^{n} \in R(\Im)$ for some positive integer $n$. Hence it follows from (1) and (QR2) that $R((f))=R\left(\left(f^{n}\right)\right) \subseteq R(\mathfrak{I})$. Now (QR1) yields $f \in R(\mathfrak{I})$. Thus $\sqrt{R(\mathfrak{I})} \subseteq R(\mathfrak{I})$. The opposite inclusion is obvious. Hence $R(\mathfrak{I})=\sqrt{R(\mathfrak{I})}$.

It follows from (QR1) and the above that $\sqrt{\mathfrak{I}} \subseteq \sqrt{R(\mathfrak{I})}=R(\mathfrak{I})$. Hence (1) and $(\mathrm{QR} 2)$ show that $R(\sqrt{\mathfrak{I}}) \subseteq R(R(\mathfrak{I}))=R(\mathfrak{I})$. The opposite inclusion follows from (1).

2. Radicals. In this section we define radical operations on the ideals of a ring (see also [E]; a special case of Theorem 2.5 is given in [B]). We show that radical operations are quasi-radical, and give a criterion for the converse to be true. From this criterion it follows that quasi-radical operations in noetherian rings are radical. 
2.1. Definition. Let $R$ be an operation on the ideals of the ring $A$. We denote by $\operatorname{Spec}(A)$ the set of prime ideals of $A$, and define

$$
\operatorname{Spec}_{R}(A)=\{\mathfrak{p} \in \operatorname{Spec}(A): R(\mathfrak{p})=\mathfrak{p}\} .
$$

We say that $R$ is a radical operation on $A$ if there is a subset $\mathcal{R}$ of $\operatorname{Spec}(A)$ such that, for all ideals $\mathfrak{I}$ of $A$,

$$
R(\mathfrak{I})=\bigcap_{\mathfrak{I} \subseteq \mathfrak{p} \in \mathcal{R}} \mathfrak{p} .
$$

In particular $R(\mathfrak{I})=A$ if there is no prime ideal $\mathfrak{p}$ in $\mathcal{R}$ such that $\mathfrak{p} \supseteq \mathfrak{I}$. The operation $R$ on the ideals of $A$ given by (2.1.1) is called the radical operation associated to $\mathcal{R}$.

Let $R$ be a radical operation on the ring $A$ and let $\mathfrak{I}$ be an ideal of $A$. We call $R(\mathfrak{I})$ the radical of $\mathfrak{I}$, and say that $\mathfrak{I}$ is radical if $R(\mathfrak{I})=\mathfrak{I}$.

2.2. EXAMPLE. (1) The well known characterization of the usual radical as the intersection of the prime ideals that contain the ideal shows that it is a radical operation.

(2) Let $\mathcal{R}$ be the subset of $\operatorname{Spec}(A)$ consisting of the maximal ideals of $A$, and let $R$ be the corresponding radical operation. For each ideal $\mathfrak{I}$ of $A$ the ideal $R(\mathfrak{I})$ is the Jacobson radical of $\mathfrak{I}$.

(3) It is shown in [BCR], [R1] and [R2] that the quasi-radical operation of Example 1.3(3) is radical.

(4) It is shown in [L1] that the quasi-radical operation of Example 1.3(4) is radical.

2.3. Proposition. Let $\mathcal{R}$ be a subset of $\operatorname{Spec}(A)$ and let $R$ be the radical operation associated to $\mathcal{R}$. Then $R$ is quasi-radical.

Moreover, $\mathcal{R} \subseteq \operatorname{Spec}_{R}(A)$, and for all ideals $\mathfrak{I}$ of $A$ we have

$$
R(\mathfrak{I})=\bigcap_{\mathfrak{I} \subseteq \mathfrak{p} \in \operatorname{Spec}_{R}(A)} \mathfrak{p} .
$$

Proof. Properties (QR1) and (QR2) are obviously satisfied, as is the inclusion $\mathcal{R} \subseteq \operatorname{Spec}_{R}(A)$. Moreover it is clear that when $\mathfrak{I}$ and $\mathfrak{J}$ are ideals of $A$ such that $\mathfrak{I} \subseteq \mathfrak{J}$ then $R(\mathfrak{I}) \subseteq R(\mathfrak{J})$. It follows that $R(\mathfrak{I} \mathfrak{J}) \subseteq R(\mathfrak{I} \cap \mathfrak{J}) \subseteq$ $R(\mathfrak{I}) \cap R(\mathfrak{J})$.

In order to prove that $R$ satisfies (QR3) it remains to prove that $R(\mathfrak{I} \mathfrak{J}) \supseteq$ $R(\mathfrak{I}) \cap R(\mathfrak{J})$. Let $f \in A \backslash R(\mathfrak{I} \mathfrak{J})$. Then there is a prime ideal $\mathfrak{p}$ in $\mathcal{R}$ such that $\mathfrak{p} \supseteq \mathfrak{I} \mathfrak{J}$, but $f \notin \mathfrak{p}$. Then at least one of the ideals $\mathfrak{I}$ and $\mathfrak{J}$ is contained in $\mathfrak{p}$. By definition $f$ is not in $R(\mathfrak{I})$ or $R(\mathfrak{J})$, and thus $f \notin R(\mathfrak{I}) \cap R(\mathfrak{J})$. Hence $R(\mathfrak{I} \mathfrak{J}) \supseteq R(\mathfrak{I}) \cap R(\mathfrak{J})$.

It remains to prove the last statement of the proposition. Since $\mathcal{R} \subseteq$ $\operatorname{Spec}_{R}(A)$ we have $\bigcap_{\mathfrak{I} \subseteq \mathfrak{p} \in \operatorname{Spec}_{R}(A)} \mathfrak{p} \subseteq \bigcap_{\mathfrak{I} \subseteq \mathfrak{p} \in \mathcal{R}} \mathfrak{p}=R(\mathfrak{I})$. To prove the opposite inclusion take an $f \in A \backslash \bigcap_{\mathfrak{I} \subseteq \mathfrak{p} \in \operatorname{Spec}_{R}(A)} \mathfrak{p}$. Then there is a prime 
ideal $\mathfrak{q} \in \operatorname{Spec}_{R}(A)$ such that $\mathfrak{q} \supseteq \mathfrak{I}$ and $f \notin \mathfrak{q}$. It follows from Proposition 1.4(1) that $\mathfrak{q}=R(\mathfrak{q}) \supseteq R(\mathfrak{I})$, and in particular $f \notin R(\mathfrak{I})$. Consequently, $R(\mathfrak{I}) \subseteq \bigcap_{\mathfrak{I} \subseteq \mathfrak{q} \in \operatorname{Spec}_{R}(A)} \mathfrak{q}$, which completes the proof.

2.4. ExAmPLE. Let $\mathcal{R}$ be the subset of $\operatorname{Spec}(A)$ consisting of the maximal ideals, and let $R$ be the radical operation associated to $\mathcal{R}$. Then an ideal $\mathfrak{I}$ of $A$ is radical if and only if it is the intersection of the maximal ideals that contain it, that is, if it is equal to its Jacobson radical. Hence the Jacobson radical is equal to the usual radical if and only if $R$ is also the radical operation associated to the set $\operatorname{Spec}(A)$ of all prime ideals of $A$.

A different proof of the last part of the following result appears in [B].

2.5. ThEOREM. Let $R$ be a quasi-radical operation on the ideals of the ring $A$. Assume that $R$ satisfies the condition

(QR4) For every family $\left\{\mathfrak{I}_{\alpha}\right\}_{\alpha \in I}$ of ideals in $A$ that is totally ordered by inclusion we have

Then $R$ is a radical operation.

$$
R\left(\bigcup_{\alpha \in I} \mathfrak{I}_{\alpha}\right)=\bigcup_{\alpha \in I} R\left(\mathfrak{I}_{\alpha}\right) .
$$

In particular, if $A$ satisfies the ascending chain condition on quasi-radical ideals, then every quasi-radical operation is radical.

Proof. Let $\mathfrak{I}$ be an ideal in $A$ and let $f \in A \backslash R(\mathfrak{I})$. In order to prove the first part of the theorem we show that there is a quasi-radical prime ideal $\mathfrak{p}$ in $A$ such that $\mathfrak{p} \supseteq \mathfrak{I}$ and $f \notin \mathfrak{p}$.

Let $\mathcal{I}$ be the set of quasi-radical ideals $\mathfrak{J}$ that contain $R(\mathfrak{I})$ but not $f$. It follows from (QR4) that for every collection $\left\{\mathfrak{J}_{\alpha}\right\}_{\alpha \in I}$ of elements in $\mathcal{I}$ that is totally ordered by inclusion, the ideal $R\left(\bigcup_{\alpha \in I} \mathfrak{J}_{\alpha}\right)=\bigcup_{\alpha \in I} R\left(\mathfrak{I}_{\alpha}\right)=\bigcup_{\alpha \in I} \mathfrak{I}_{\alpha}$ is an upper bound of $\mathcal{I}$. Hence by Zorn's Lemma there is a maximal element $\mathfrak{p}$ in $\mathcal{I}$.

We show that $\mathfrak{p}$ is a prime ideal. Let $g$ and $h$ be elements of $A \backslash \mathfrak{p}$. Assume that $g h \in \mathfrak{p}$. Since $\mathfrak{p}$ is maximal in $\mathcal{I}$ by assumption, it follows from (QR2) that $f \in R(A g+\mathfrak{p})$ and $f \in R(A h+\mathfrak{p})$. Property (QR3) shows that $R((A g+\mathfrak{p})(A h+\mathfrak{p}))=R(A g+\mathfrak{p}) \cap R(A h+\mathfrak{p})$. However, $(A g+\mathfrak{p})(A h+\mathfrak{p}) \subseteq \mathfrak{p}$ since we have assumed that $g h \in \mathfrak{p}$, and by Proposition 1.4(1) we obtain $R((A g+\mathfrak{p})(A h+\mathfrak{p})) \subseteq R(\mathfrak{p})=\mathfrak{p}$, and hence $R(A g+\mathfrak{p}) \cap R(A h+\mathfrak{p}) \subseteq \mathfrak{p}$. In particular, $f \in \mathfrak{p}$, which contradicts $\mathfrak{p} \in \mathcal{I}$. Hence $\mathfrak{p}$ is a prime ideal, and $\mathfrak{p} \supseteq \mathfrak{I}, f \notin \mathfrak{p}$.

To prove the final assertion of the theorem we show that when $A$ satisfies the ascending chain condition for quasi-radical ideals, then (QR4) holds. By (QR1) and (QR2), we always have $R\left(\bigcup_{\alpha \in I} \Im_{\alpha}\right) \supseteq \bigcup_{\alpha \in I} R\left(\Im_{\alpha}\right)$ and $\bigcup_{\alpha \in I} \Im_{\alpha} \subseteq R\left(\bigcup_{\alpha \in I} \Im_{\alpha}\right)$. When $A$ satisfies the ascending chain condition for quasi-radical ideals there is an element $\beta$ of $I$ such that $\bigcup_{\alpha \in I} R\left(\mathfrak{I}_{\alpha}\right)=$ 
$R\left(\mathfrak{I}_{\beta}\right)$. In particular $\bigcup_{\alpha \in I} \mathfrak{I}_{\alpha} \subseteq R\left(\mathfrak{I}_{\beta}\right)$, and thus $R\left(\bigcup_{\alpha \in I} \mathfrak{I}_{\alpha}\right) \subseteq R R\left(\mathfrak{I}_{\beta}\right)=$ $R\left(\mathfrak{I}_{\beta}\right)=\bigcup_{\alpha \in I} R\left(\mathfrak{I}_{\alpha}\right)$. Hence (QR4) holds.

2.6. Remark. Condition (QR4) clearly holds for the usual radical. Hence the well known result, mentioned in Example 2.2(1), that $\sqrt{\mathfrak{I}}$ is the intersection of the prime ideals that contain $\mathfrak{I}$ is a very particular case of Theorem 2.5 .

(QR4) holds for all the quasi-radicals of Example 1.3. Hence the assertions of Example 2.2 that all these quasi-radical operations are radical follow from Theorem 2.5.

2.7. Example. From Proposition 2.3 it follows that a quasi-radical operation on $A$ does not necessarily satisfy (QR4). We shall give an example showing that even radical operations do not necessarily have this property.

Let $A$ be a local ring with maximal ideal $\mathfrak{m}$, and assume that there is a chain of prime ideals $\mathfrak{p}_{1} \subseteq \mathfrak{p}_{2} \subseteq \cdots$ in $A$, each properly contained in $\mathfrak{m}$, such that $\bigcup_{i=1}^{\infty} \mathfrak{p}_{i}=\mathfrak{m}$. Let $R$ be the radical operation on $A$ associated to the set $\mathcal{R}=\{\mathfrak{p}: \mathfrak{p} \neq \mathfrak{m}\}$. Then $R(\mathfrak{m})=A$, and thus $R\left(\bigcup_{i=1}^{\infty} \mathfrak{p}_{i}\right)=A$, while $\bigcup_{i=1}^{\infty} R\left(\mathfrak{p}_{i}\right)=\bigcup_{i=1}^{\infty} \mathfrak{p}_{i}=\mathfrak{m}$.

An example of a ring $A$ with the above properties is the polynomial ring $K\left[t_{1}, t_{2}, \ldots\right]$ in the variables $t_{1}, t_{2}, \ldots$ over a field $K$, localized at the maximal ideal $\left(t_{1}, t_{2}, \ldots\right)$ with $\mathfrak{p}_{i}=\left(t_{1}, \ldots, t_{i}\right) K\left[t_{1}, t_{2}, \ldots\right]_{\left(t_{1}, t_{2}, \ldots\right)}$.

3. Quasi-radicals on topological spaces. In this section we introduce quasi-radical operations on closed subsets of a topological space. We show that the particular operation that to each closed set associates the closure of its interior is quasi-radical.

3.1. Notation. Let $X$ be a topological space. For every subset $W$ of $X$ we denote by $\bar{W}$ the closure of $W$ in $X$, and by $W^{0}$ the interior of $W$.

3.2. Definition. Let $X$ be a topological space. An operation $Q$ on the closed subsets of $X$ is a correspondence that to every closed subset $Y$ of $X$ associates a closed subset $Q(Y)$ of $X$. We write

$$
X_{Q}=\{x \in X: Q(\overline{\{x\}})=\overline{\{x\}}\} .
$$

The operation $Q$ is quasi-radical if it satisfies the following three conditions for all closed subsets $Y$ and $Z$ of $X$ :

$(\mathrm{QR} 1) \quad Q(Y) \subseteq Y$.

(QR2) $\quad Q(Q(Y))=Q(Y)$.

(QR3) $\quad Q(Y \cup Z)=Q(Y) \cup Q(Z)$.

3.3. EXAMPLES. The identity operation that associates every closed subset of $X$ to itself is clearly a quasi-radical operation on the closed subsets of $X$. It is the finest quasi-radical operation on the closed subsets. 
The coarsest operation on the closed subsets of $X$ is the operation $Q$ defined by $Q(Y)=\emptyset$ for all closed subsets $Y$ of $X$.

In Proposition 3.6 we shall show that the operation that to every closed subset associates the closure of its interior is a quasi-radical operation.

3.4. Lemma. Let $X$ be a topological space and let $Y$ and $Z$ be closed subsets such that $Z \subseteq Y$. For every quasi-radical operation $Q$ on the topological space $X$ we have $Q(Z) \subseteq Q(Y)$.

Proof. It follows from (QR3) that if $Z \subseteq Y$, then $Q(Y)=Q(Y \cup Z)=$ $Q(Y) \cup Q(Z)$ and hence $Q(Z) \subseteq Q(Y)$.

3.5. Lemma. Let $X$ be a topological space and let $Y$ and $Z$ be closed subsets. Then

(1) $\overline{Y^{0}} \subseteq Y$.

(2) $\left(\overline{Y^{0}}\right)^{0}=Y^{0}$.

(3) $\overline{Y^{0} \cup Z^{0}}=\overline{(Y \cup Z)^{0}}$.

Proof. (1) Clearly $Y^{0} \subseteq Y$, and thus $\overline{Y^{0}} \subseteq \bar{Y}=Y$.

(2) We have $Y^{0} \subseteq \overline{Y^{0}}$, and $Y^{0}$ is open in $X$. Consequently, $Y^{0} \subseteq\left(\overline{Y^{0}}\right)^{0}$. Conversely, it follows from (1) that $\overline{Y^{0}} \subseteq \bar{Y}=Y$, and thus $\left(\overline{Y^{0}}\right)^{0} \subseteq Y^{0}$. Hence $\left(\overline{Y^{0}}\right)^{0}=Y^{0}$.

(3) It is clear that $Y^{0} \cup Z^{0} \subseteq(Y \cup Z)^{0}$. Hence $\overline{Y^{0} \cup Z^{0}} \subseteq \overline{(Y \cup Z)^{0}}$.

The opposite inclusion follows from $(Y \cup Z)^{0} \subseteq \overline{Y^{0} \cup Z^{0}}$, which we now prove. Let $x \in(Y \cup Z)^{0}$. Then every neighborhood of $x$ contains an open neighborhood $U$ of $x$ such that $U \subseteq Y \cup Z$. We shall distinguish three cases:

(i) If $U \subseteq Y \cap Z$ we have $U \subseteq Y^{0} \cap Z^{0}$, and in particular $U \cap\left(Y^{0} \cup Z^{0}\right) \neq \emptyset$.

(ii) If $U \cap(Y \backslash Z) \neq \emptyset$ then $U_{1}=U \cap(X \backslash Z)=U \cap(Y \backslash Z)$ is an open non-empty subset of $U$ and $U_{1}=U \cap(X \backslash Z) \subseteq(Y \cup Z) \cap(X \backslash Z) \subseteq Y$. Hence $U_{1} \subseteq Y^{0}$, and we obtain $U \cap\left(Y^{0} \cup Z^{0}\right) \neq \emptyset$.

(iii) If $U \cap(Z \backslash Y) \neq \emptyset$ we find, as in part (ii), that $U \cap\left(Y^{0} \cup Z^{0}\right) \neq \emptyset$.

In all three cases $U \cap\left(Y^{0} \cup Z^{0}\right) \neq \emptyset$ and thus every neighborhood of $x$ intersects $Y^{0} \cup Z^{0}$. Hence $x \in \overline{Y^{0} \cup Z^{0}}$, and so $(Y \cup Z)^{0} \subseteq \overline{Y^{0} \cup Z^{0}}$ as desired.

3.6. Proposition. Let $X$ be a topological space. For every closed subset $Y$ of $X$ we let

$$
Q(Y)=\overline{Y^{0}}
$$

Then $Q$ defines a quasi-radical operation on the closed subsets of $X$.

Proof. (1) It follows from Lemma 3.5(1) that $Q(Y)=\overline{Y^{0}} \subseteq Y$. Hence (QR1) is fulfilled.

(2) It follows from Lemma 3.5(2) that $Q(Q(Y))=\overline{\left(\overline{Y^{0}}\right)^{0}}=\overline{Y^{0}}=Q(Y)$. Hence (QR2) is fulfilled. 
(3) It follows from Lemma 3.5(3) that $Q(Y \cup Z)=\overline{(Y \cup Z)^{0}}=\overline{Y^{0} \cup Z^{0}}$. We have $\overline{Y^{0} \cup Z^{0}}=\overline{Y^{0}} \cup \overline{Z^{0}}$, and thus $Q(Y \cup Z)=Q(Y) \cup Q(Z)$. That is, (QR3) is satisfied.

4. Radicals on topological spaces. In this section we define radical operations on closed subsets of topological spaces and show that radical operations are quasi-radical. We give a criterion for topological spaces that guarantees that the quasi-radical operation that associates to every closed set the closure of its interior is not radical. Moreover we give an example of a topological space that satisfies this criterion.

4.1. Definition. Let $X$ be a topological space and let $Q$ be an operation on the closed subsets of $X$. We say that $Q$ is a radical operation if there is a subset $W$ of $X$ such that for all closed subsets $Y$ of $X$ we have

$$
Q(Y)=\overline{Y \cap W}
$$

We call $Q$ the radical operation associated to $W$.

4.2. Lemma. Let $X$ be a topological space and let $V, W \subseteq X$. Then

$$
\overline{\overline{V \cap W} \cap W}=\overline{V \cap W} .
$$

In particular, for every point $x$ in $W$, we have

$$
\overline{\overline{\{x\}} \cap W}=\overline{\{x\}} .
$$

Proof. We have $V \cap W \subseteq \overline{V \cap W} \cap W$, and thus $\overline{V \cap W} \subseteq \overline{\overline{V \cap W} \cap W}$. Conversely, the inclusion $\overline{V \cap W} \cap W \subseteq \overline{V \cap W}$ gives $\overline{\overline{V \cap W} \cap W} \subseteq \overline{\overline{V \cap W}}$ $=\overline{V \cap W}$.

4.3. Proposition. Let $X$ be a topological space and let $Q$ be the radical operation associated to a subset $W$ of $X$. Then $Q$ is a quasi-radical operation on closed subsets of $X$. Moreover, $W \subseteq X_{Q}$, and for all closed subsets $Y$ of $X$ we have

$$
Q(Y)=\overline{Y \cap X_{Q}}
$$

Proof. Let $Y$ and $Z$ be closed subsets of $X$.

(1) We have $Q(Y)=\overline{Y \cap W} \subseteq \bar{Y}=Y$. Hence (QR1) holds for $Q$.

(2) It follows from Lemma 4.2 that $Q(Q(Y))=\overline{\overline{Y \cap W} \cap W}=\overline{Y \cap W}=$ $Q(Y)$. Hence (QR2) holds for $Q$.

(3) We have $Q(Y \cup Z)=\overline{(Y \cup Z) \cap W}=\overline{(Y \cap W) \cup(Z \cap W)}=\overline{Y \cap W} \cup$ $\overline{Z \cap W}=Q(Y) \cup Q(Z)$. Hence (QR3) holds for $Q$.

We now prove the last part of the proposition. Let $x \in W$. It follows from Lemma 4.2 that $Q(\overline{\{x\}})=\overline{\{x\}} \cap W=\overline{\{x\}}$, and hence $x \in X_{Q}$. Thus $W \subseteq X_{Q}$ and so $Q(Y)=\overline{Y \cap W} \subseteq \overline{Y \cap X_{Q}}$. In order to prove the opposite inclusion let $x \in Y \cap X_{Q}$. By definition of $X_{Q}$ we have $\overline{\{x\}}=Q(\overline{\{x\}})=$ 
$\overline{\overline{\{x\}} \cap W} \subseteq \overline{\bar{Y} \cap W}=\overline{Y \cap W}$. Hence $x \in \overline{Y \cap W}$ and so $Y \cap X_{Q} \subseteq \overline{Y \cap W}=$ $Q(Y)$. Together with the inclusion $Q(Y) \subseteq \overline{Y \cap X_{Q}}$ proved above we have $Q(Y)=\overline{Y \cap X_{Q}}$, as desired.

4.4. Lemma. Let $X$ be a topological space that has the following two properties:

(i) For all points $x \in X$ we have $\overline{\{x\}}^{0}=\emptyset$.

(ii) $X=X_{1} \cup X_{2}$, where $X_{1}$ and $X_{2}$ are open and closed subsets of $X$ both different from $X$.

Then the quasi-radical operation $Q$ that associates to a closed set $Y$ the closure of its interior $\overline{Y^{0}}$ is not radical. More precisely, $X_{Q}=\{x \in X$ : $Q(\overline{\{x\}})=\overline{\{x\}}\}=\emptyset$, so $\overline{X_{i} \cap X_{Q}}=\emptyset$, and $Q\left(X_{i}\right)=X_{i}$ for $i=1,2$.

Proof. By (i), $Q(\overline{\{x\}})=\overline{\overline{\{x\}}^{0}}=\emptyset$ for every $x \in X$. Hence $X_{Q}=\emptyset$. Consequently, Proposition 4.3 shows that if $Q$ were a radical operation then $Q(Y)=\overline{Y \cap \emptyset}=\emptyset$ for all closed subsets $Y$ of $X$. However, (ii) yields $Q\left(X_{i}\right)=$ $\overline{X_{i}^{0}}=X_{i}$ for $i=1,2$.

4.5. Example. We now give an example of a topological space that satisfies conditions (i) and (ii) of Lemma 4.4. In particular we obtain an example showing that not all quasi-radical operations on topological spaces are radical. More precisely, the quasi-radical operation defined in Proposition 3.6 is not always radical.

Let $X_{1}$ and $X_{2}$ be two disjoint sets each with infinitely many elements. It is easily checked that all the subsets of $X=X_{1} \cup X_{2}$ that consist of all but a finite number of points of one of the sets $X, X_{1}$ or $X_{2}$, together with the empty set, form the open sets of a topology on $X$. In this topology conditions (i) and (ii) are clearly fulfilled.

5. Radicals for the Zariski topology. In this section we investigate quasi-radical and radical operations on the closed sets of the spectrum of a ring with the Zariski topology. We show that the correspondence between ideals in the ring and the closed subsets of the spectrum gives a correspondence between quasi-radical and radical operations on the ideals of the ring, and the quasi-radical and radical operations on the closed subsets of the spectrum.

5.1. Notation. Let $A$ be a ring and let $X=\operatorname{Spec}(A)$ be the collection of prime ideals of $A$. In order to distinguish elements in $X$ from the prime ideals in $A$ we write $\mathfrak{p}_{x}$ for the prime ideal in $A$ corresponding to the element $x$ in $X$. For every ideal $\mathfrak{I}$ in $A$ we write

$$
\mathcal{V}(\mathfrak{I})=\left\{x \in X: \mathfrak{p}_{x} \supseteq \mathfrak{I}\right\}=\bigcup_{\mathfrak{p}_{x} \supseteq \mathfrak{I}}\{x\},
$$


and for every subset $W$ of $X$ we write

$$
\mathcal{I}(W)=\left\{f \in A: f \in \mathfrak{p}_{x} \text { for all } x \in W\right\}=\bigcap_{x \in W} \mathfrak{p}_{x} .
$$

5.2. Remark. The sets $\mathcal{V}(\mathfrak{I})$ for all ideals $\mathfrak{I}$ in $A$ are the closed subsets of the Zariski topology on $X$. Clearly the following properties hold for all ideals $\mathfrak{I}$ and $\mathfrak{J}$ of $A$, all closed subsets $Y$ and $Z$ of $X$, and all subsets $W$ of $X$ (see e.g. $[\mathrm{H}])$ :

(1) $\mathcal{I}(\mathcal{V}(\mathfrak{I}))=\sqrt{\mathfrak{I}}$.

(2) $\mathcal{V}(\mathcal{I}(Y))=Y$.

(3) $\mathcal{V}(\mathfrak{I} \mathfrak{J})=\mathcal{V}(\mathfrak{I} \cap \mathfrak{J})=\mathcal{V}(\mathfrak{I}) \cup \mathcal{V}(\mathfrak{J})$ and $\mathcal{I}(Y \cup Z)=\mathcal{I}(Y) \cap \mathcal{I}(Z)=$ $\sqrt{\mathcal{I}(Y) \mathcal{I}(Z)}$

(4) $\mathcal{V}\left(\mathfrak{p}_{x}\right)=\overline{\{x\}}, \mathcal{I}(\overline{\{x\}})=\mathfrak{p}_{x}$ and $\mathcal{I}(W)=\mathcal{I}(\bar{W})$.

5.3. Theorem. Let $A$ be a ring and let $X=\operatorname{Spec}(A)$ have the Zariski topology. Denote by $Q$ a quasi-radical operation on the closed subsets of $X$. The operation $R$ on the ideals of $A$ that to an ideal $\mathfrak{I}$ associates the ideal

$$
R(\mathfrak{I})=\mathcal{I}(Q(\mathcal{V}(\mathfrak{I})))
$$

is a quasi-radical operation on the ideals of $A$. Moreover,

$$
X_{Q}=\operatorname{Spec}_{R}(A),
$$

and $Q$ is a radical operation on the closed subsets of $X$ if and only if $R$ is a radical operation on the ideals of $A$.

Proof. (1) It follows from (QR1) for $Q$ that $R(\mathfrak{I})=\mathcal{I}(Q(\mathcal{V}(\mathfrak{I}))) \supseteq$ $\mathcal{I}(\mathcal{V}(\mathfrak{I}))$. Moreover, property (1) of Remark 5.2 implies that $\mathcal{I}(\mathcal{V}(\mathfrak{I})) \supseteq \mathfrak{I}$. Hence $R(\mathfrak{I}) \supseteq \mathfrak{I}$, and so (QR1) holds for $R$.

(2) It follows from property (2) of Remark 5.2 that

$$
R(R(\mathfrak{I}))=\mathcal{I}(Q(\mathcal{V}(\mathcal{I}(Q(\mathcal{V}(\mathfrak{I}))))))=\mathcal{I}(Q(Q(\mathcal{V}(\mathfrak{I})))) .
$$

Moreover, $(\mathrm{QR} 2)$ for $Q$ yields $\mathcal{I}(Q(Q(\mathcal{V}(\mathfrak{I}))))=\mathcal{I}(Q(\mathcal{V}(\mathfrak{I})))=R(\mathfrak{I})$. Hence (QR2) holds for $R$.

(3) From property (3) of Remark 5.2 it follows that

$$
R(\mathfrak{I} \cap \mathfrak{J})=\mathcal{I}(Q(\mathcal{V}(\mathfrak{I} \cap \mathfrak{J})))=\mathcal{I}(Q(\mathcal{V}(\mathfrak{I} \mathfrak{J})))=R(\mathfrak{I} \mathfrak{J}),
$$

and $R(\mathfrak{I} \cap \mathfrak{J})=\mathcal{I}(Q(\mathcal{V}(\mathfrak{I} \cap \mathfrak{J})))=\mathcal{I}(Q(\mathcal{V}(\mathfrak{I}) \cup \mathcal{V}(\mathfrak{J})))$. Moreover, (QR3) for $Q$ and property (3) of Remark 5.2 yield

$$
\begin{aligned}
\mathcal{I}(Q(\mathcal{V}(\mathfrak{I}) \cup \mathcal{V}(\mathfrak{J}))) & =\mathcal{I}(Q(\mathcal{V}(\mathfrak{I})) \cup Q(\mathcal{V}(\mathfrak{J})))=\mathcal{I}(Q(\mathcal{V}(\mathfrak{I}))) \cap \mathcal{I}(Q(\mathcal{V}(\mathfrak{J}))) \\
& =R(\mathfrak{I}) \cap R(\mathfrak{J}) .
\end{aligned}
$$

Hence (QR3) holds for $R$. We have thus shown that $R$ is a quasi-radical operation on the ideals of $A$.

We now prove the second part of the theorem. 
Let $x \in X_{Q}$, that is, $Q(\overline{\{x\}})=\overline{\{x\}}$. We deduce from (4) of Remark 5.2 that $Q\left(\mathcal{V}\left(\mathfrak{p}_{x}\right)\right)=\overline{\{x\}}$, and so $R\left(\mathfrak{p}_{x}\right)=\mathcal{I}\left(Q\left(\mathcal{V}\left(\mathfrak{p}_{x}\right)\right)\right)=\mathcal{I}(\overline{\{x\}})=\mathfrak{p}_{x}$. Consequently, $x \in \operatorname{Spec}_{R}(A)$. Thus $X_{Q} \subseteq \operatorname{Spec}_{R}(A)$.

Conversely, let $x \in \operatorname{Spec}_{R}(A)$. It follows from (4) of Remark 5.2 that $\mathfrak{p}_{x}=$ $R\left(\mathfrak{p}_{x}\right)=\mathcal{I}\left(Q\left(\mathcal{V}\left(\mathfrak{p}_{x}\right)\right)\right)=\mathcal{I}(Q(\overline{\{x\}}))$. Consequently, by (4) of Remark 5.2, $\overline{\{x\}}=\mathcal{V}\left(\mathfrak{p}_{x}\right)=\mathcal{V}\left(R\left(\mathfrak{p}_{x}\right)\right)=\mathcal{V}(\mathcal{I}(Q(\overline{\{x\}})))=Q(\overline{\{x\}})$. That is, we have $x \in X_{Q}$, and so $\operatorname{Spec}_{R}(A) \subseteq X_{Q}$. Thus $X_{Q}=\operatorname{Spec}_{R}(A)$. It follows that for every ideal $\mathfrak{I}$ of $A$ we have

$$
\mathcal{I}\left(\mathcal{V}(\mathfrak{I}) \cap X_{Q}\right)=\mathcal{I}\left(\mathcal{V}(\mathfrak{I}) \cap \operatorname{Spec}_{R}(A)\right)=\bigcap_{x \in \mathcal{V}(\mathfrak{I}) \cap \operatorname{Spec}_{R}(A)} \mathfrak{p}_{x} .
$$

Assume that $R$ is a radical operation on the ideals of $A$. It follows from Proposition 2.3 that $R(\mathfrak{I})=\bigcap_{x \in \mathcal{V}(\mathfrak{I}) \cap \operatorname{Spec}_{R}(A)} \mathfrak{p}_{x}$ for all ideals $\mathfrak{I}$ of $A$. Hence we infer from (5.3.1) that $R(\mathfrak{I})=\mathcal{I}\left(\mathcal{V}(\mathfrak{I}) \cap X_{Q}\right)$. It follows from (4) of Remark 5.2 that $\mathcal{I}\left(\mathcal{V}(\mathfrak{I}) \cap X_{Q}\right)=\mathcal{I}\left(\overline{\mathcal{V}(\mathfrak{I}) \cap X_{Q}}\right)$. Thus $\mathcal{I}(Q(\mathcal{V}(\mathfrak{I})))=R(\mathfrak{I})=$ $\mathcal{I}\left(\overline{\mathcal{V}(\mathfrak{I}) \cap X_{Q}}\right)$. From $(2)$ of Remark 5.2 we thus obtain

$$
Q(\mathcal{V}(\mathfrak{I}))=\mathcal{V}(\mathcal{I}(Q(\mathcal{V}(\mathfrak{I}))))=\mathcal{V}(R(\mathfrak{I}))=\mathcal{V}\left(\mathcal{I}\left(\overline{\mathcal{V}(\mathfrak{I}) \cap X_{Q}}\right)\right)=\overline{\mathcal{V}(\mathfrak{I}) \cap X_{Q}}
$$

for all ideals $\mathfrak{I}$ of $A$. Consequently, the operation $Q$ is radical.

Conversely, assume that $Q$ is radical. It follows from Proposition 4.3 that $Q(\mathcal{V}(\mathfrak{I}))=\overline{\mathcal{V}(\mathfrak{I}) \cap X_{Q}}$ for all ideals $\mathfrak{I}$ of $A$. Hence (5.3.1) and property (4) of Lemma 5.2 show that

$$
R(\mathfrak{I})=\mathcal{I}(Q(\mathcal{V}(\mathfrak{I})))=\mathcal{I}\left(\overline{\mathcal{V}(\mathfrak{I}) \cap X_{Q}}\right)=\mathcal{I}\left(\mathcal{V}(\mathfrak{I}) \cap X_{Q}\right)=\bigcap_{x \in \mathcal{V}(\mathfrak{I}) \cap \operatorname{Spec}_{R}(A)} \mathfrak{p}_{x} .
$$

Consequently, the operation $R$ is radical, and we have proved the theorem.

5.4. EXAMPLE. We shall give an example of a ring with a quasi-radical operation that is not radical.

It follows from Proposition 3.6 and from Theorem 5.3 that for every ring $A$ we have a quasi-radical operation $R$ on the ideals of $A$ that associates to an ideal $\mathfrak{I}$ of $A$ the ideal

$$
R(\mathfrak{I})=\mathcal{I}\left(\overline{\mathcal{V}(\mathfrak{I})^{0}}\right) .
$$

If the Zariski topology of $\operatorname{Spec}(A)$ satisfies conditions (i) and (ii) of Lemma 4.4 then Lemma 4.4 and Theorem 5.3 show that $R$ is not radical.

We next construct a ring $A$ such that $\operatorname{Spec}(A)$ with the Zariski topology satisfies conditions (i) and (ii) of Lemma 4.4. Let $K\left[t_{1}, t_{2}, \ldots\right]$ be the polynomial ring in the countable number of independent variables $t_{1}, t_{2}, \ldots$ over a field $K$, and let $A$ be the residue ring of $K\left[t_{1}, t_{2}, \ldots\right]$ by the ideal $\mathfrak{I}$ generated by the elements $t_{i}\left(t_{i}-1\right)$ for $i=1,2, \ldots$. We denote by $u_{i}$ the residue class of $t_{i}$ in $A$ for $i=1,2, \ldots$ Every prime ideal in $A$ contains $u_{i}$ or $u_{i}-1$ for each $i=1,2, \ldots$. Consequently, every prime ideal is of the 
form $\left(u_{1}-\delta_{1}, u_{2}-\delta_{2}, \ldots\right)$, where $\delta_{i}$ is equal to 0 or 1 , and clearly every ideal of this form is maximal. In particular all points of $\operatorname{Spec}(A)$ are closed.

Let $f\left(t_{1}, \ldots, t_{n}\right)$ be an element of $\mathfrak{I}$. Then $f\left(\delta_{1}, \ldots, \delta_{n}\right)=0$ for all choices of $\delta_{1}, \ldots, \delta_{n}$. Conversely, it is easily shown by induction on $n$ that if $f\left(\delta_{1}, \ldots, \delta_{n}\right)=0$ for all choices of $\delta_{1}, \ldots, \delta_{n}$ then $f\left(t_{1}, \ldots, t_{n}\right)$ is in $\mathfrak{I}$. In particular, for every $f\left(u_{1}, \ldots, u_{n}\right) \neq 0$ in $A$ we can find $\delta_{1}, \ldots, \delta_{n}$ such that $f\left(\delta_{1}, \ldots, \delta_{n}\right) \neq 0$. Since $f\left(u_{1}, \ldots, u_{n}\right)$ is in $\left(u_{1}-\delta_{1}, u_{2}-\delta_{2}, \ldots\right)$ if and only if $f\left(\delta_{1}, \ldots, \delta_{n}\right)=0$ we see that if $f\left(u_{1}, \ldots, u_{n}\right) \neq 0$ then $f\left(u_{1}, \ldots, u_{n}\right)$ is not in $\left(u_{1}-\delta_{1}, u_{2}-\delta_{2}, \ldots\right)$ for any choices of $\delta_{n+1}, \delta_{n+2}, \ldots$. Consequently, if $f\left(u_{1}, \ldots, u_{n}\right) \neq 0$ then $X \backslash \mathcal{V}\left(f\left(u_{1}, \ldots, u_{n}\right)\right)$ contains infinitely many points of $X$. It is well known that the open sets $X \backslash \mathcal{V}\left(f\left(u_{1}, \ldots, u_{n}\right)\right)$ form a basis for the Zariski topology of $X$. Hence every non-empty open subset of $\operatorname{Spec}(A)$ contains infinitely many points. In particular, the points of $\operatorname{Spec}(A)$ cannot be open. Since they are closed it follows that $\overline{\{x\}}^{0}=\{x\}^{0}=\emptyset$, that is, condition (i) of Lemma 4.4 is satisfied.

To prove that condition (ii) of Lemma 4.4 is also satisfied we observe that the closed subsets $X_{1}=\mathcal{V}\left(u_{1}\right)$ and $X_{2}=\mathcal{V}\left(u_{1}-1\right)$ of $X$ are disjoint and $X=X_{1} \cup X_{2}$, hence they are also open.

In the ring $A$ with the radical operation (5.4.1) the ideals $\left(u_{1}\right)$ and $\left(u_{1}-1\right)$ are both examples of ideals that are quasi-radical, but not the intersection of radical primes. To see this we note that it follows from the definition of the quasi-radical operation on $A$ that $R\left(u_{1}\right)=\mathcal{I}\left(Q\left(\mathcal{V}\left(u_{1}\right)\right)\right)=\mathcal{I}\left(Q\left(X_{1}\right)\right)$, and that the last part of Lemma 4.4 shows that $Q\left(X_{1}\right)=X_{1}$. Therefore $R\left(u_{1}\right)=\mathcal{I}\left(X_{1}\right)$ and thus $\mathcal{I}\left(X_{1}\right)$ is the intersection of all ideals of the form $\left(u_{1}, u_{2}-\delta_{2}, u_{3}-\delta_{3}, \ldots\right)$. As observed above, this intersection is equal to $\left(u_{1}\right)$. Hence $R\left(u_{1}\right)=\left(u_{1}\right)$, that is, the ideal $\left(u_{1}\right)$ is quasi-radical for the operation $R$. However, the ideal $\left(u_{1}\right)$ is not the intersection of radical primes, because, as we have seen, the set of such primes is empty.

\section{References}

[BGN] E. Becker, R. Grobe and M. Niermann, Radicals of binomial ideals, J. Pure Appl. Algebra 117/118 (1997), 41-79.

[B] A. Benhissi, Hilbert's basis theorem for $*$-radical ideals, ibid. 161 (2001), 245-253.

[BCR] J. Bochnak, M. Coste et M.-F. Roy, Géométrie algébrique réelle, Ergeb. Math. Grenzgeb. 12, Springer, Berlin, 1987.

[E] L. Ernström, The spectrum associated to a radical operation, Math. Scand. 73 (1993), 5-14.

[H] R. Hartshorne, Algebraic Geometry, Grad. Texts in Math. 52, Springer, New York, 1977.

[K1] W. Krull, Jacobsonsche Ringe, Hilbertscher Nullstellensatz, Dimensionstheorie, Math. Z. 54 (1951), 354-387. 
[K2] W. Krull, Jacobsonsches Radikal und Hilbertscher Nullstellensatz, in: Proc. Internat. Congress of Mathematicians (Cambridge, MA, 1950), Vol. 2, Amer. Math. Soc., Providence, RI, 1952, 56-64.

[L1] D. Laksov, Generalized radicals of ideals in algebras, Conference on Algebraic Varieties of Small Dimension (Turin, 1985), Rend. Sem. Mat. Univ. Politec. Torino 1986, Special Issue (1987), 77-89.

[L2] - Radicals and Hilbert Nullstellensatz for not necessarily algebraically closed fields, Enseign. Math. 33 (1987), 323-338.

[R1] J.-J. Risler, Un théorème des zéros en géométrie analytique réelle, C. R. Acad. Sci. Paris Sér. A-B 274 (1972), A1488-A1490.

[R2] - Le théorème des zéros en géométries algébrique et analytique réelles, Bull. Soc. Math. France 104 (1976), 113-127.

[R] M. Rosenlund, Radical operations in rings and topological spaces, thesis, Royal Technical Institute of Stockholm, 2004.

Department of Mathematics

Royal Technical Institute

S-100 44 Stockholm, Sweden

E-mail: laksov@math.kth.se

mro@math.kth.se

Received 3 November 2004;

in revised form 24 January 2005 